\title{
A study of Dhat syndrome- a culture bound syndrome in Nepalese context
}

\author{
Dhungana $\mathbf{M}^{1}$, Ghimire $\mathrm{SR}^{2}$, Thapa $\mathbf{M}^{3}$, Kafle $\mathrm{B}^{1}$
}

1. Lecturer, Department of Psychiatry, Devdaha Medical College, Rupandehi, Nepal 2. Consultant Psychiatrist and Project Manager, Transcultural Psychosocial Organization Nepal (TPO Nepal), Kathmandu, Nepal. 3. Consultant Psychiatrist, Chautari Nepal Health Foundation, Rupandehi, Nepal

E-mail *Corresponding author: drdhungana3536@hotmail.com

\begin{abstract}
Introduction: Dhat syndrome is generally believed to be a culture-bound which is characterized by excessive preoccupation with loss of "Dhat," which is generally taken to be representing semen. The study evaluated the symptoms of patient with Dhat syndrome visiting three mental health clinics of Rupandehi district.
\end{abstract}

Material And Method This descriptive cross- sectional study was conducted in three different Psychiatry outpatient clinics at Rupandehi district of Nepal. Purposive sampling, a type of non-random sampling was utilized for the study. A total 50 patients with Dhat syndrome were enrolled in the study. A semi-structured Performa containing socio-demographic and Dhat syndrome symptoms related information was filled by respondents after providing written informed consent.

Results: The patients were predominantly young adults, male with mean age of $23.1 \pm 4.6$. Majority of them were in age group 18-25 years, hindu by religion, living in non-urban areas, family income of 5000-10000 per month, literate and unmarried. A range of psychological and somatic symptoms associated with Dhat syndrome were revealed. Most common symptoms found in the study were excessive worries (96\%), tingling sensation of body $(86 \%)$, weakness $(80 \%)$, decrease interest $(80 \%)$, fatigue $(76 \%)$, depressed mood $(74 \%)$, and generalized body ache $(72 \%)$.

Conclusion: Dhat syndrome presented with a range of psychological and somatic symptoms. Any male patient presenting with multiple somatic symptoms must be evaluated for Dhat syndrome and the physician must enquire about the semen loss and the associated beliefs.

Keywords: Culture Bound Syndrome, Dhat Syndrome, Semen Loss

\section{INTRODUCTION}

The term Dhat syndrome was first used by Wig in 1960 to describe a culture bound sexual neurosis, for patients preoccupied with the excessive loss of semen by nocturnal emissions or in urine. ${ }^{1}$ Both ICD-10 Classification of Mental and Behavioural Disorders (ICD-10) 2 and Diagnostic and statistical manual of mental disorders (DSM-5) ${ }^{3}$ classified Dhat syndrome as neurotic disorder and culture specific Disorder respectively relating to loss of semen. Several studies from pointed out Dhat syndrome as a widely recognized condition from the Indian subcontinent that is associated with fatigue and pre-occupation with semen loss as the main complaint. ${ }^{4-11}$ Most of the studies related to Dhat 
syndrome have been done in India, however, its concept has been also described in western cultures. ${ }^{5}$ Apart from Indian studies, a study done in Pakistan reported that about $30 \%$ of the subjects complained of Dhat and the prevalence of Dhat was equal in patients with functional and organic diagnosis. ${ }^{12}$ Similarly another study done on treatment seeking pattern of subjects with Dhat syndrome in Lahore found out about $50 \%$ of the subjects sought help from traditional healer (Hakims). ${ }^{13}$ In studies from Sri Lanka, Dhat syndrome has been reported in many patients, of them approximately half had somatic symptoms. ${ }^{14,15}$

Patients with Dhat syndrome present a range of vague symptoms of worries, generalized weakness, bodily ache, fatigue, palpitations, loss of interest, headaches, dizziness, sadness. ${ }^{9,16}$ Recent study by Grover and his colleagues 10 revealed range of psychological and somatic symptoms in patients with Dhat syndrome that include weakness; low energy; feeling down, depressed or hopeless; lack of interest or pleasure in doing things; mental weakness; anger; irritability; excessive worry; pain in arms, legs or joints (knees, hips, etc.), and disturbed sleep. This study aims to find out psychological and somatic symptoms experienced by patient with Dhat syndrome. The belief may also be accompanied by anxiety or depressive symptoms and the patient may present with or without sexual dysfunction. ${ }^{17}$

\section{MATERIAL AND METHOD}

This descriptive cross- sectional study was conducted in three different Psychiatry outpatient clinics at Rupandehi district of Nepal. The study sample comprised a total of 50 male patients. Purposive sampling, a type of nonrandom sampling was utilized for the study. The male patients of age of 18 years and above presenting in our clinics with symptoms consistent with Dhat syndrome were screened by pre-defined criteria. Patients who fulfilled the ICD-10 2 diagnosis of Dhat syndrome were included in the study. Exclusion criteria of the study were existence of mental retardation /intellectual disability, psychotic illness, organic mental disorder, and physical cause for discharge per urethra. After obtaining written informed consent from participants, semistructured Performa which covered the sociodemographic and Dhat syndrome related questionnaire was provided to all participants. Data were entered manually into statistical package for social science (SPSS-20) and then it was analyzed by using descriptive statistics in term of frequency and percentage. Interpretation of the study was done on the basis of analyzed data using tables.

\section{RESULT}

The sample consisted of 50 male patients with mean age of $23.1 \pm 4.6$. Table 1 explored the socio-demographic profile of respondents in which majority of them were in age group 18-25 years, Hindu by religion, living in non-urban areas, family income of 5000-10000 per month, literate and unmarried.

Table 1: Socio-Demographic profile of study sample

\begin{tabular}{|l|c|}
\hline Variables & $\mathbf{N}(\%)$ \\
Age & $20(40 \%)$ \\
18-25 yrs & $17(34 \%)$ \\
$26-35$ yrs & $8(16 \%)$ \\
36-45yrs & $5(10 \%)$ \\
>45yrs & $40(80 \%)$ \\
Religion & $5(10 \%)$ \\
Hindu & $4(8 \%)$ \\
Muslim & $1(2 \%)$ \\
Buddhist & \\
Christian & $3(6 \%)$ \\
Educational Status & $8(16 \%)$ \\
Illiterate & $13(26 \%)$ \\
Primary school & $15(30 \%)$ \\
High school & $11(22 \%)$ \\
Intermediate & \\
Graduate and/or above & $9(18 \%)$ \\
Living areas & $41(82 \%)$ \\
Urban & \\
Non-urban & $8(16 \%)$ \\
Family income & $28(56 \%)$ \\
Below NRs 5000 & $14(28 \%)$ \\
NRs. 5000-10000 & \\
More than 10000 & $29(58 \%)$ \\
Marital Status & $21(42 \%)$ \\
Unmarried & \\
Married & \\
\hline
\end{tabular}


Table 2 presented a range of psychological and somatic symptoms associated with Dhat syndrome. Most common symptoms found in study were excessive worries (96\%), tingling sensation of body ( $86 \%)$, weakness ( $80 \%)$, decrease interest $(80 \%)$, fatigue $(76 \%)$, depressed $\operatorname{mood}(74 \%)$, and generalized body ache $(72 \%)$.

Table 2: Distribution of symptoms associated with Dhat Syndrome

\begin{tabular}{|l|c|}
\hline Symptoms & $\mathbf{N}(\%)$ \\
Excessive worries & $48(96 \%)$ \\
Weakness & $40(80 \%)$ \\
Fatigue & $38(76 \%)$ \\
Generalized body ache & $36(72 \%)$ \\
Pain arms, legs, joints & $29(58 \%)$ \\
Back pain & $26(52 \%)$ \\
Stomach ache & $24(48 \%)$ \\
Restlessness & $30(60 \%)$ \\
Palpitation & $19(38 \%)$ \\
Sweating & $21(42 \%)$ \\
Throat discomfort & $22(44 \%)$ \\
Headache & $31(62 \%)$ \\
Depressed mood & $37(74 \%)$ \\
Feeling of guilt & $16(32 \%)$ \\
Tingling sensation of body & $43(86 \%)$ \\
Anorexia & $12(24 \%)$ \\
Sleep disturbances & $31(62 \%)$ \\
Dizziness & $24(48 \%)$ \\
Shortness of breath & $11(22 \%)$ \\
Chest pain & $18(36 \%)$ \\
Decrease interest & $40(80 \%)$ \\
Nausea or vomiting & $16(32 \%)$ \\
Altered bowel habit & $9(18 \%)$ \\
Poor concentration & $31(62 \%)$ \\
Burning sensation of urine & $26(52 \%)$ \\
Anger and irritability & $24(48 \%)$ \\
& \\
\hline
\end{tabular}

\section{DISCUSSION:}

To the best of our knowledge, this is the first study on patients of Dhat syndrome from Nepal. Dhat syndrome forms an important health problem especially of adolescent and young male populations of Asian region. ${ }^{19}$ Although Dhat syndrome has been included in nosological classification (ICD-10), ${ }^{2}$ associated psychological and somatic symptoms are lagging on manuals. ${ }^{10}$ Demographic findings of the study show that approximately above two-third of respondent were age group of 18 to 35 years and were married. Various past studies suggested that Dhat syndrome is usually seen in young, unmarried, or recently married men with conservative attitude toward sex. ${ }^{10,11}$ This study revealed that most of respondents (82\%) were Hindu and 12\% were Muslim. This is because of presence predominant Hindu population in this locality. Other reason being more Hindu participants in this study might be because of treatment seeking behavior among these populations of this region. This finding is concurrence with the past studies finding in similar settings. ${ }^{10,19}$

Existing researches suggest that Dhat syndrome is more common in those residing in rural areas. ${ }^{19,} 20$ Findings of the present study also support the same in which $82 \%$ respondents were living in non-urban areas. Low education was found to be associated with Dhat syndrome in many past studies which was also in concordance with our study findings. However, some studies found Dhat syndrome among people with all educational level. ${ }^{10}$ Further study in diverse population with an appropriate sample size could explore the association of Dhat syndrome and education level. Majorities of respondents' (72\%) family income in our study was less than 10,000 rupees a month and had low socioeconomic status. Past studies revealed concordance findings and have reported that patients with Dhat syndrome more commonly belonged to lower socioeconomic status. ${ }^{19,} 20$ In contrast, Grover and his colleagues found Dhat syndrome among people belonging to middle socioeconomic status..$^{10}$

There is clear evidence that patients with Dhat syndrome have various accompanying psychological and somatic symptoms. ${ }^{10,20,21}$ In present study, there are list of symptoms that include various symptoms of depression, anxiety and somatoform disorder.

The most commonly noted psychological and somatic symptoms in the present study include excessive worries, weakness, fatigue, generalized body ache, tingling sensation of body parts, depressed mood, decrease interest, poor concentration and headache apart from 
range of symptoms. Our study findings were consistent with other studies findings in similar settings. Similar symptoms associated with Dhat syndrome were demonstrated by past studies. Worries being the most reported symptoms in our study which was also reported by patient with hat syndrome in a past study in similar setting. ${ }^{16}$ A phenomenology study of Dhat syndrome revealed range of common symptoms as Sense of being unhealthy, worry, and feeling that there will be no improvement despite treatment, tension, tiredness , fatigue , weakness, and anxiety . ${ }^{11}$ Similarly, another large study among Indian population found out bodily weakness; feeling tired or having low energy; feeling down, depressed or hopeless; low interest or pleasure in doing things; mental weakness; excessive worry; pain in arms, legs or joints (knees, hips, etc.), and trouble sleeping among majorities of patients. ${ }^{10}$

Considering all past studies including our study, it is clear that a number of psychological and somatic symptoms were exhibited by patient with Dhat syndrome. Detail evaluation for co-morbidities such as depression, anxiety disorder, somatoform disorder and psychosexual disorder is essential to decrease associated morbidity and mortality. Presence of sexual co-morbidity is quite common among patients with Dhat syndrome. ${ }^{10}$ However; our study did not include sexual related symptoms among study participants.

The findings our study is limited by the number of symptoms evaluated and limited sample size. The symptoms of patient with Dhat syndrome obtained as part of this study was limited to the content of the questionnaire used. It is quite possible that the questionnaire may not include some of the symptoms. The study was not based on use of qualitative method of assessment. The use of qualitative methods could have expanded the list further for assessment of various symptoms. Sample was taken from patients seeking clinical consultations at mental health clinics; therefore, results could not be generalized to other group of patients.

\section{CONCLUSION:}

Dhat syndrome presented with a range of psychological and somatic symptoms. Any male patient presenting with multiple somatic symptoms must be evaluated for Dhat syndrome and the physician must enquire about the semen loss and the associated beliefs.

\section{CONFLICT OF INTEREST:}

The authors report no conflicts of interest in this work, and are solely responsible for the content and writing of this paper. No funding has been received for this work.

\section{REFERENCES:}

1. Tseng WS. From peculiar psychiatric disorders through culture-bound syndromes to culturerelated specific syndromes. Transcult Psychiatry, 2006; 43; 554-576.

2. World Health Organization. The ICD-10 Classification of Mental and Behavioural Disorders: Clinical Descriptions and Diagnostic Guidelines. Geneva: World Health Organization, 1992.

3. American Psychiatric Association. Diagnostic and statistical manual of mental disorders: DSM5. Washington, D.C: American Psychiatric Association, 2013.

4. Ranjith, G. and Mohan, R. Dhat syndrome as a functional somatic syndrome: developing a sociosomatic model. Psychiatry, 2006; 69(2):14250 .

5. Sumathipala A, Siribaddana S, Bhugra D. Culture - bound syndromes: The story of dhat syndrome. Br J Psychiatry' 2004;184:2009.

6. Bhatia M, Choudhary S. Dhat Syndrome culture bound sex neurosis. Indian J Medical Sci, 1998; 52: 30-5.

7. Perme B, Ranjith G, Mohan R et al. Dhat (semen loss) syndrome: a functional somatic syndrome of the Indian subcontinent? Gen Hosp Psychiatry, 2005; 27(3): 215-17.

8. Rajkumar RP, Bharadwaj B. Dhat syndrome: Evidence for a depressive spectrum subtype. Asian Journal of Psychiatry, 2014; 9:57-60.

9. Deb KS, Balhara YS. Dhat syndrome: A review of the world literature. Indian Journal of Psychological Medicine, 2013; 35:326-31.

10. Grover S, Avasthi A, Gupta S, Neogi R, Behere $P B$, et al. Phenomenology and beliefs of patients with Dhat syndrome: A nationwide multicentric study. Int J Soc Psychiatry; 2016;62(1):57-66.

11. Prakash S, Sharan P, Sood M. A study on phenomenology of Dhat syndrome in men in a general medical setting. Indian J Psychiatry, 2016;58:129-41

12. Mumford DB. The Dhat syndrome: A culturally determined symptom of depression? Acta Psychiatrica Scandinavica, 1996;94:163-7.

13. Khan N. Dhat syndrome in relation to demographic characteristics. Indian Journal of Psychiatry, 2005;47: 54-7. 
14. De Silva P, Dissanayake SAW. The use of semen syndrome in Sri Lanka: A clinical study. Sexual $\mathcal{E}$ Marital Therapy, 1989;4: 195-204.

15. Dewaraja $R \mathcal{E}$ Sasaki $Y$ (1991). Semen loss syndrome: A comparison between Sri Lanka and Japan. American Journal of Psychotherapy, 1991; 45: 14-20.

16. Prakash O. Lessons for postgraduate trainees about Dhat syndrome. Indian J Psychiatry, 2007;49:208-10.

17. Grover S, Dutt A, Avasthi A. Culture bound syndrome. In: Vyas JN $\mathcal{E}$ Ghimire SR, editors. Text Book of Postgraduate Psychiatry, New Delhi, Jaypee Brothers Medical Publishers; 2016.

18. Bhatia MS, Malik SC. Dhat syndrome18. Sahu RN, Sharma VK, Kumar A, Bavishi C, Balaji $M$. Dhat syndrome and its association with sexual behavior and pyschiatric co-morbidities in males: a case control study. Int J Med Res Heath Sci. 2015;4(1):90-3.

19. Behere PB, Natraj GS. Dhat syndrome: The phenomenology of a culture-bound sex neurosis of the orient. Indian Journal of Psychiatry, 1984;26:76-8.

20. Grover S, Avasthi A, Aneja J, Shankar G, Mohan MR, Nehra $R, \mathcal{E}$ Padhy $S$. Comprehensive questionnaire for assessment of Dhat syndrome: Development and use in patient population. Journal of Sexual Medicine, 2014;11:2485-95. 